\title{
Offset transients modulate attentional capture by sudden onsets
}

\author{
ROBIN MARTIN-EMERSON and ARTHUR F. KRAMER \\ University of Illinois at Urbana-Champaign, Urbana, Illinois
}

\begin{abstract}
Recent research with visual search tasks has suggested that stimuli which appear as sudden onsets (new objects) have attentional priority over stimuli that are created by removing segments of premasks (non-onset stimuli). Attentional capture by sudden onsets occurs despite the fact that the appearance of these new objects predicts neither the identity nor the location of the target in the visual search task. In three experiments, we examined the extent to which attentional capture by sudden onsets could be modulated by offset transients used to create non-onset objects. To that end, we systematically manipulated the ratio of non-onset to onset stimuli in the display (display ratio) as well as the ratio of offset to onset segments between the stimulus types (stimulus ratio). Increases in either the stimulus ratio or the display ratio resulted in increases in the visual search slopes for the onset targets. These results suggest that the ability of sudden onsets (new objects) to capture attention is influenced by stimulusdriven factors, such as environmental change. Interestingly, the results also indicated that goal-directed or purposeful search for sudden-onset (new-object) targets was relatively uninfluenced by the amount of change in the visual display. Therefore, it would appear that environmental change has differential effects on goal-directed and stimulus-driven search. These results are discussed in terms of their implications for our understanding of attentional capture.
\end{abstract}

A central premise of theories of visual selective attention is that attention can be directed or guided by both goaldirected and stimulus-driven components (Treisman \& Sato, 1990; Wolfe, 1994). In a visual search task, a defining characteristic of the target may serve to direct attention in a top-down, or goal-directed, manner. Salient local differences between stimuli can also guide attention in a bottom-up, or stimulus-driven, fashion.

In recent years, the bottom-up, or stimulus-driven, component of visual attention has been studied in the form of a phenomenon referred to as attentional capture. Attentional capture has typically been studied in multiple-item displays in which one item possesses a unique attribute that is irrelevant to the visual search task. That is, the unique attribute predicts neither the identity nor the location of the target. Attentional capture is said to occur if reaction time (RT) is independent of display size when the unique item serves as the target. In contrast, responses to nonunique targets increase in latency with increasing display size.

In Yantis and Jonides's (1984; Jonides \& Yantis, 1988) experiments, an onset stimulus presented in a display of

This research was supported by Grant N00014-92-J-1792 from the Office of Naval Research, Grant R01AG12203 from the National Institutes of Health, and Cooperative Agreement DAAL01-96-2-0003 with the Army Research Laboratory. Portions of this research were reported at the 1995 Annual Meeting of the Human Factors and Ergonomics Society in San Diego. The authors would like to thank Chip Folk, Jan Theeuwes, Steve Yantis, and Mike Braunstein for their insightful comments and suggestions on an earlier version of this paper. Correspondence should be addressed to A. F. Kramer, Beckman Institute, University of Illinois, 405 North Mathews Ave., Urbana, IL 61801 (e-mail: akramer@s.psych.uiuc.edu). one or three non-onset stimuli was shown to capture attention. In their visual search task, the stimuli were initially presented as an array of seven-segment figure-eight premasks. The letters of the search display were formed by removal of two segments from each premask (i.e., non-onset stimuli). An additional five-segment letter appeared in a previously blank location (i.e., an onset stimulus). The onset stimulus occurred as the target letter on $1 / d$ trials, with $d$ defined as the number of items in the display. Therefore, the fact that one stimulus occurred as an onset was not predictive of target identity or location. RT to onset target letters was independent of display size, whereas RTs to non-onset targets increased with display size. Yantis and Jonides interpreted these findings as evidence that attention is captured by onsets such that onset stimuli are attended first when they occur among nononset stimuli.

In contrast to onsets, other irrelevant feature singletons (i.e., a stimulus that differs from others along a single dimension), such as color (Jonides \& Yantis, 1988; Theeuwes, 1990; Todd \& Kramer, 1994), form (Theeuwes, 1990), and luminance (Jonides \& Yantis, 1988; Todd \& Kramer, 1994; Yantis \& Hillstrom, 1994), do not appear to capture attention. Yantis and his colleagues have interpreted these findings as evidence that onsets are unique in their ability to capture attention (but see Folk, Remington, \& Johnston, 1992). Specifically, Yantis and Hillstrom postulated that, rather than discontinuities in luminance produced by onsets, the appearance of a new perceptual object in the visual field captures attention in a stimulusdriven manner. Further support for the new-object interpretation was provided by Hillstrom and Yantis (1994), who demonstrated that motion per se fails to capture at- 
tention. Only when motion serves to segregate an object from the visual field will attention be captured, because motion then characterizes a stimulus as a new object.

There is, however, research that suggests that onsetsand, therefore, the simple appearance of new objectsare not alone in their ability to capture attention (Miller, 1989; Theeuwes, 1990,1991). Miller noted that the onset stimulus used by Yantis and Jonides (1984; Jonides \& Yantis, 1988) produced a greater number of segment transients than did a non-onset stimulus formed from a premask. Miller speculated that the relationship between offset and onset transients could influence attentional capture. In his study, Miller constructed complex premasks composed of a sufficient number of segments such that the number of segment transients was approximately equal between a non-onset and an onset stimulus. Miller observed an increase in RT between display sizes of 2 and 4 that did not differ for onset and non-onset targets. He concluded that the transient change of non-onsets may also capture attention, although to a lesser degree than onsets (i.e., new objects).

Theeuwes $(1990,1991)$ also considered transient change to be a factor in stimulus-driven allocation of attention. The effect of a change to a single stimulus was explored in a study (Theeuwes, 1990) using a display consisting of 4,8 , or 16 line segments, each within a surrounding form. The display included one unique form (either a circle among diamonds or a diamond among circles) that changed into a nonunique form $260 \mathrm{msec}$ after display onset. In the experimental condition, the location and identity of a target line segment were uncorrelated with the changing form; in the control condition, the target always appeared in the changing form.

Theeuwes (1990) observed a small display size effect (slope $=5.9 \mathrm{msec} /$ item) in the control condition indicating that when the change in form was perfectly correlated with the target line segment, subjects were able to effectively allocate attention on the basis of a stimulus transient. RT to a target appearing within the changing form was slower in the experimental condition relative to the control condition, but it was faster than targets that appeared in an unchanged form. Theeuwes estimated that capture by the change in form occurred on approximately $25 \%$ of the unique trials.

In a subsequent study, Theeuwes (1991) extended Miller's (1989) work by providing additional evidence that transients (i.e., offsets as well as onsets) modulate the control of attention. Theeuwes provided a $100 \%$ valid central cue at 600 or $300 \mathrm{msec}$ prior to or $200 \mathrm{msec}$ after the display of four stimuli. A peripheral offset uncorrelated with the target location was also presented 160 or $80 \mathrm{msec}$ prior to, concurrently with, or $80 \mathrm{msec}$ after the display of four stimuli. In the absence of a focus of attention at the target location (central cue SOA $200 \mathrm{msec}$ ), RT was faster when the peripheral offset occurred at target locations rather than at nontarget locations, indicating that peripheral offsets captured attention.

Prior research has shown that when attention is focused at a location other than that where an onset stimulus subsequently occurs (Theeuwes, 1991; Yantis \& Jonides, 1990 ) or when an attentional set has been adopted (Folk et al., 1992), onsets fail to capture attention. These results indicate the attentional capture by new objects may be overridden by top-down, or goal-directed, processing. The findings of Miller (1989) and Theeuwes $(1990,1991)$ suggest that, unlike merely salient stimulus attributes such as color and luminance, which do not typically capture attention (Jonides \& Yantis, 1988; Theeuwes, 1990; Todd \& Kramer, 1994; but see Folk et al., 1992), offset transients may attract attention. Thus, it would appear plausible that bottom-up, or stimulus-driven, processing of transient offsets may also modulate the extent to which sudden onsets, or new objects, capture attention. Both goal-directed and stimulus-driven factors may impact the process of attentional capture by sudden-onset stimuli.

How might the processing of transient offsets modulate attentional capture by new objects? This question can be considered within the context of a model of attentional priority proposed by Yantis and colleagues (Yantis \& Hillstrom, 1994; Yantis \& Johnson, 1990; Yantis \& Jones, 1991). Within this model, attention is directed to objects on the basis of their relative priority, which is established, on a moment-by-moment basis, by both goaldirected (e.g., knowledge about object characteristics, such as form, color, or location) and stimulus-driven (e.g., perceptual grouping or new objects) factors. Yantis and colleagues further suggest that stimulus-driven selection is mediated by an interrupt that occurs whenever a new object appears in the visual field. The interrupt serves to enhance the priority of the new object, which, in turn, increases the probability that this object will be processed early during visual search.

We hypothesize that the mechanism responsible for the generation of attentional interrupts is susceptible to noise that results from changes in the physical characteristics of old (non-onset) objects. Furthermore, we suggest that the magnitude of the noise increases with the amount of display change. Taken to its logical conclusion, such a noisy interrupt mechanism would, with large amounts of display change, direct attention in a relatively random fashion to objects in the visual field. This follows if we assume that interrupts are generated whenever activation exceeds some absolute threshold. Thus, due to a decreasing signal-to-noise ratio for the onset with increasing display change, non-onset objects would begin to capture attention on an increasing proportion of trials. However, such an outcome appears unlikely given previous findings of relatively steep search slopes for non-onset stimuli (Miller, 1989; Theeuwes, 1990).

Instead, it would appear more likely that, with increasing display change, onset objects would fail to capture attention on a larger proportion of trials but non-onset objects would continue to be searched in a serial selfterminating manner. This type of outcome would occur if a proportion, or ratio, threshold was the basis for the generation of an attentional interrupt. That is, if we assume that (1) the interrupt mechanism is susceptible to noise associated with display change, (2) the generation 
of an attentional interrupt occurs when the activation provided by the onset exceeds the activation attributed to noise by a fixed proportion, and (3) the activation associated with the appearance of an onset or new object is a stochastic process, then the proportion of trials on which an attentional interrupt is elicited by new objects would decrease with increases in the magnitude of display (nononset) change. This follows, since it would become increasingly difficult to exceed the fixed proportional threshold difference between signal (i.e., generated by the appearance of the onset) and noise (i.e., produced by offset transients that create non-onset objects) with increasing display change. Of course, the ratio threshold rule would also serve to prevent the elicitation of interrupts to non-onset objects, since the likelihood of activation associated with any stimulus exceeding threshold declines with an increasing magnitude of display change. Miller's (1989) findings are consistent with such a proposal: The search slope for the onset targets approached that of the non-onset targets when the number of transient segments were equated for the onset and non-onset stimuli. Henceforth, we will refer to our proposal that the capture of attention by new objects is modulated by non-onset display change as the interrupt threshold hypothesis.

We examined the interrupt threshold hypothesis in three experiments by orthogonally manipulating the ratio of non-onset to onset stimuli in the display (display ratio) and the ratio of offset to onset segments in individual stimuli (stimulus ratio). Both of these factors influence the amount of non-onset change relative to onset change in the display and therefore are expected to modulate the degree to which onset targets capture attention. Thus, according to the interrupt threshold hypothesis, we would expect less capture by the onset targets, as indexed by increases in the slope of the visual search function, with increases in the display and the stimulus ratio.

In Experiment 1, we manipulated the display ratio by varying the number of non-onset stimuli that appeared in the display. We found that attentional capture decreased for onset targets with increases in the number of non-onset stimuli in the display. However, in a control condition in which subjects specifically searched for an onset stimulus, RT was relatively unaffected by the number of nononset stimuli appearing in the display. In Experiment 2, we manipulated the display ratio and the stimulus ratio by increasing the complexity of the non-onset premasks (see Figure 1), which resulted in an increase in the number of premask offsets used to form non-onset stimuli relative to Experiment 1 . In this case, we found relatively steep search slopes for onset targets even when few nononset stimuli appeared in the display. Finally, in Experiment 3 , we further increased the complexity of the nononset premasks and found an additional increase in the search slope for the onset targets. When viewed together, these results are consistent with the interrupt threshold hypothesis. Capture of attention by sudden onsets, as reflected by the slope of the visual search function, is modulated by the amount of display change.

\section{EXPERIMENT 1}

To examine the extent to which offset transients modulate capture by irrelevant onsets (i.e., onsets that predict neither the identity nor the location of a target), four display sizes were used in a visual search task modeled after Yantis and Jonides (1984; Jonides \& Yantis, 1988). In each display, non-onset stimuli were created by removing the appropriate segments of simple figure-eight premasks to form letters, and an irrelevant onset letter appeared in a previously blank location. In the onsetirrelevant condition, to test the hypothesis that onsets capture attention independent of task relevance, the frequency of an onset target was constrained to preclude the possibility of onset predicting target location.

With few stimuli in the display, the ratio of non-onset stimuli to onset stimuli is small and approximates that of previous studies. Consequently, we expect to observe attentional capture (i.e., relatively shallow search slopes) at small display sizes. Note, however, that as display size increases, the display ratio also increases, and our interrupt threshold hypothesis predicts that capture of attention by the onset stimulus should become less likely, as reflected in an increase in the search slope when larger numbers of non-onset stimuli are present in the display.

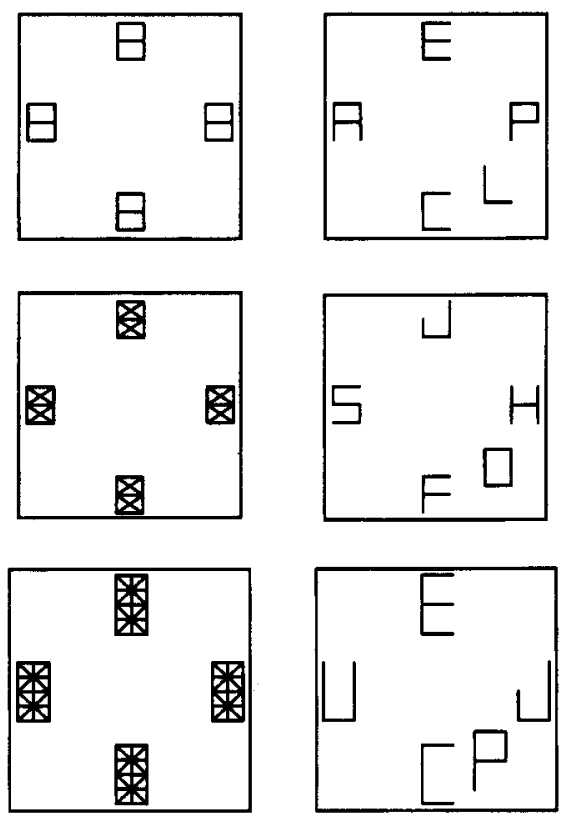

Figure 1. The premask displays (left column) used to form the non-onset letters and the stimulus displays (right column) for each experiment. Non-onset letters were formed by removing the appropriate segments from the figure eights (Experiment 1, top panels), the figure eights with an $X$ inscribed in each half of the figure (Experiment 2, center panels), or the figure eights with a four-segment star inscribed in each half of the figure (Experiment 3, bottom panels). The onset letter appeared in a previously blank location. The height-to-width ratio of the premasks and letters reflects the relative size of the stimuli, but note that the displays are not drawn to scale. 
The attentional priority model proposed by Yantis and colleagues (Yantis \& Hillstrom, 1994; Yantis \& Johnson, 1990; Yantis \& Jones, 1991) suggests that the appearance of an onset, or new object, in the display results in the production of an attentional interrupt, which, in turn, leads to the early identification of the onset stimulus. Since the attentional interrupt is thought to be insensitive to the number of non-onset stimuli in the visual field, this model does not predict a change in the slope of the search function for the onset targets with changes in display size.

With an increase in display size and a constant eccentricity of stimuli, the visual angle between adjacent stimuli decreases. Therefore, it is conceivable that factors affecting stimulus salience, such as contour interference or perceptual grouping of the stimuli, could preclude capture by an irrelevant onset. To test for this possibility, a between-subjects design was implemented. In the onsetrelevant condition, when present, the target was always the onset letter. A shallow display size slope for the onsetrelevant target condition would suggest that stimulus proximity does not preclude localizing an onset target in the onset-irrelevant condition.

\section{Method}

Subjects. Sixteen University of Illinois undergraduate students were paid for their participation in the experiment. Eight subjects ( 3 women and $5 \mathrm{men}$ ) participated in the onset-irrelevant condition, and 8 subjects ( 4 women and 4 men) participated in the onsetrelevant condition. All subjects demonstrated adequate near and far visual acuity (minimum 20/40) with the Snellen eye chart.

Apparatus and Stimuli. The stimuli were presented on an Amdek AM/815E SVGA monitor and driven by a Zeos $486(33 \mathrm{MHz})$ personal computer. The refresh rate of the graphics screen was $90 \mathrm{~Hz}$. Subject responses were collected with an IBM AT keyboard.

The stimulus array was initially displayed as a set of sevensegment, block figure-eight ("8") premasks equally spaced about a virtual circle. The top panels of Figure 1 illustrate the figure-eight premask display and the stimulus display of letters formed by the offset of the appropriate segments from each premask. The stimulus set consisted of 11 block letters (A, C, E, F, H, J, L, O, P, S, and $U)$. As the non-onset stimulus letters were formed, the onset letter appeared in a previously blank location on the virtual circle, equidistant from adjacent non-onset stimuli. A stimulus display of 3, 5, 7 , or 13 letters then consisted of the letters formed by a set of 2,4 , 6 , or 12 premasks, respectively, and a single onset letter. At a viewing distance of $90 \mathrm{~cm}$, each premask or letter appeared at $4.5^{\circ}$ from the circle's center and subtended $0.6^{\circ}$ of visual angle in height and $0.5^{\circ}$ in width.

Across the four display sizes, there were 24 possible positions that a stimulus could occupy. In the 3-letter display, two premasks were positioned opposite one another on the virtual circle, yielding six possible premask configurations. Multiple premask configurations allowed stimulus location to vary from trial to trial. In each configuration for the 3-letter display, the onset stimulus occurred at one of two previously blank locations, equidistant from adjacent non-onset stimuli. The 5-, 7-, and 13-letter displays were similarly constructed, such that the premasks were equally spaced about the virtual circle, and the onset stimulus occurred at a previously blank position equidistant from adjacent non-onset stimuli. Thus, for a specified display size, the separation between a non-onset and an onset stimulus remained constant. The minimum edge-to-edge separation (occurring at display size 13) between non-onset and onset stimuli was $0.5^{\circ}$ of visual angle. The white stimuli had luminance of $13.0 \mathrm{~cd} / \mathrm{m}^{2}$ on a black background of $0.1 \mathrm{~cd} / \mathrm{m}^{2}$.
Design. A $2 \times 2 \times 4$ design was used to manipulate onset (relevant or irrelevant) between subjects; target type (target present or absent) and display size (3, 5, 7, or 13 letters) were varied within subjects. An equal number of trials was presented for each display size. On half the trials, the target was present; on the remaining half, the target was absent. For each display size, selection of one of the possible stimulus array configurations was random, subject to the requirement that each configuration was used with equal frequency. Each letter of the stimulus set served as a target and as the onset stimulus with approximately equal frequency. With the exception of display size 13 , no letter was repeated in a display.

In the onset-irrelevant condition, the target was not correlated with the onset stimulus. Thus, an onset target occurred on $1 / d$ of the targetpresent trials, in which $d$ equals display size. A non-onset target occurred on the remaining target-present trials. In the onset-relevant condition, when present, the target was always an onset stimulus.

The subjects in the onset-irrelevant condition participated in two, approximately $1-h$, sessions. The onset-relevant condition was conducted in a single session. Each session consisted of 11 blocks of 96 trials each. The first block of trials was considered practice and was excluded from analysis, yielding a total of 1,920 experimental trials per subject for the onset-irrelevant condition and 960 trials per subject for the onset-relevant condition.

Procedure. Each trial began with the presentation of a target letter in the center of the display. The subjects pressed the space bar of the keyboard provided for response input to extinguish the display of the target letter; $500 \mathrm{msec}$ later, the premasks were displayed around a center fixation cross. After $1,000 \mathrm{msec}$, segments of the premasks were removed to form the non-onset letters, and the onset letter simultaneously appeared in a previously blank location. The stimulus letters were displayed until the subject's response.

For half of the subjects, one key (F) was used to respond to the presence of a target, and a second key $(J)$ was used to respond to the absence of a target. For the remaining half of the subjects, the response keys were reversed. RT was recorded from the display of the stimulus letters until the trial was terminated by the subject's response. Two types of errors were possible: either an incorrect response or an irrelevant response produced by striking a key other than the two designated keys. A brief tone was emitted by the computer when either error occurred. The subject's response was followed by the display of the target letter for the next trial. At the conclusion of each block of trials, a performance graph displayed average accuracy and RT information to the subject.

In the onset-relevant condition, the appearance of the onset letter and its correlation with the target were described to the subjects. No information regarding the onset letter was provided to subjects in the onset-irrelevant condition. All subjects were instructed to respond as quickly as possible while attempting to minimize errors.

\section{Results and Discussion}

Prior to analysis, observations with an RT less than $100 \mathrm{msec}$ or greater than 3 standard deviations above a subject's mean in each experimental condition were excluded. Irrelevant responses (i.e., when subjects pressed a key without a designated response) were also excluded from the analysis. These procedures removed less than $2 \%$ of the data.

Mean RTs for correct trials (collapsed across sessions) as a function of display size are plotted by target type for the onset-irrelevant condition and the onset-relevant condition in the top and bottom panels, respectively, of Figure 2. Error rates in all the experimental conditions (collapsed across sessions) are presented in Table 1. We first report separate analyses of variance (ANOVAs) for the onset-irrelevant and onset-relevant data and then describe 
the results of the ANOVAs that contrast the RTs and error rates for the onset trials in the onset-irrelevant and onsetrelevant conditions.

Onset-irrelevant condition. The mean RTs and error rate data were submitted to three-way, repeated measures ANOVAs with session ( 1 and 2$)$, display size $(3,5,7$, and 13), and target type (target present and target absent) as factors. RTs were slower in the first session than in the second session $[F(1,7)=6.70, p<.05]$. As shown in Figure 2 , RT increased with display size $[F(3,21)=48.53$, $p<.01]$ and between target-present and target-absent conditions $[F(1,7)=28.46, p<.01]$. Display size and target type interacted such that the target-absent trials showed greater increases in RT with increasing display size than did the target-present trials $[F(3,21)=27.69, p<.01]$.

Error rate increased across display size $[F(3,21)=26.13$, $p<.01]$ and between the target-absent and target-present conditions $[F(1,7)=140.26, p<.01]$. Display size interacted with session $[F(3,21)=4.80, p<.01]$, with a larger increase in error rate observed for Session 2 with increasing display size.

A second ANOVA was conducted to further examine the two target-present conditions, with session ( $\mathrm{l}$ and 2 ),
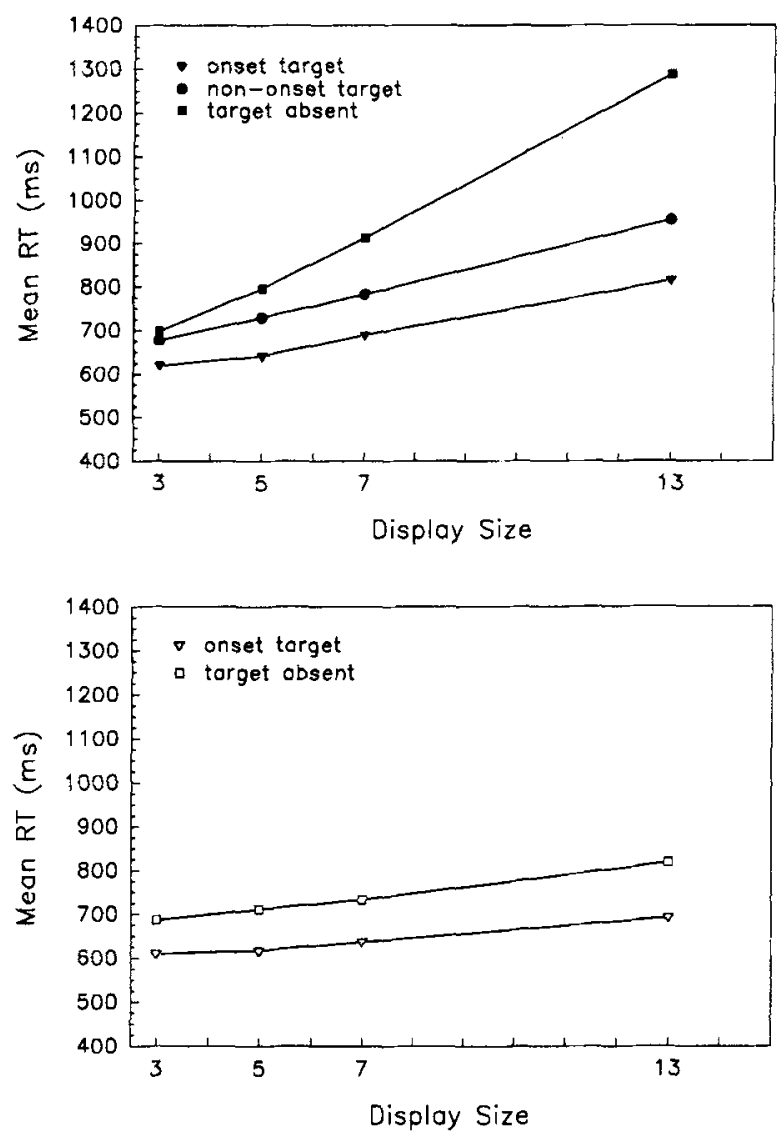

Figure 2. Mean RT (in milliseconds) by display size for onset target, non-onset target, and target-absent trials in the onsetirrelevant (top panel) and onset-relevant (bottom panel) conditions of Experiment 1.
Table 1

Error Rate for Display Size (DS) by Target Type in the Onset-Irrelevant and Onset-Relevant Conditions of Experiment 1

\begin{tabular}{lcccc} 
& \multicolumn{4}{c}{ Error Rate } \\
\cline { 2 - 5 } \multicolumn{1}{c}{ Target } & DS3 & DS5 & DS7 & DS13 \\
\cline { 3 - 5 } Absent & \multicolumn{3}{c}{ Onset Irrelevant } & \\
Non-onset & .10 & .05 & .05 & .09 \\
Onset & .06 & .16 & .15 & .20 \\
& & .09 & .08 & .11 \\
Absent & .05 & .05 & .06 & .07 \\
Onset & .10 & .08 & .09 & .09 \\
\hline
\end{tabular}

display size $(3,5,7$, and 13$)$, and target type (onset and non-onset) as factors. RT decreased between sessions $[F(1,7)=7.31, p<.05] . \mathrm{RT}$ increased with increasing display size $[F(3,21)=38.42, p<.01]$ and between onset and non-onset targets $[F(1,7)=37.37, p<.01]$. Most importantly, a significant interaction was obtained between display size and target type $[F(3,21)=6.91, p<.01]$.

According to the interrupt threshold hypothesis, if non-onsets modulate the capture of attention by onsets, then we would expect to observe a slower search rate for onset targets in large displays than in small displays. As illustrated in Figure 2 (see also Table 2), the search rate was relatively fast for the onset targets in the small displays. However, the search rate doubled for the onset targets in the larger displays. On the other hand, the search rate for non-onset targets was relatively stable across display sizes.

To evaluate the display size and target type interaction, an analysis of simple effects was conducted for target type between adjacent display sizes. The main effects of display size and target type were significant between each pair of adjacent display sizes $(p s<.01)$. The display size $x$ target type interaction was significant between the smallest display sizes $[F(1,7)=9.44, p<.05]$. However, the interaction was not statistically significant for the larger display sizes $(p s>.10)$.

The error rate in the onset-irrelevant condition increased between onset and non-onset targets $[F(1,7)=$ $25.31, p<.01]$ and as display size increased $[F(3,21)=$ $9.59, p<.01]$.

These findings suggest a modulating effect of nononsets upon attentional capture by an irrelevant onset, consistent with our hypothesis. While Miller's (1989) study demonstrated that the relationship between the transients of a non-onset stimulus and the transients of an onset stimulus influences capture by new objects, our results indicate that the display ratio, the number of non-onset stimuli relative to an onset stimulus, also affects the ability of new objects to capture attention. At larger display sizes, with a sufficient number of non-onset stimuli, the effectiveness of onsets to capture attention is reduced.

Onset-relevant condition. The possible influence of increasing proximity of adjacent stimuli with increasing display size was examined in the onset-relevant condition when the presence or absence of a target was perfectly cor- 
Table 2

Search Slope (Milliseconds/ltem) and Intercept (Milliseconds) for the Target-Present Conditions of Experiment 1

\begin{tabular}{|c|c|c|c|c|c|}
\hline \multirow[b]{2}{*}{ Target } & \multicolumn{4}{|c|}{ Search Slope } & \multirow{2}{*}{$\frac{\text { Intercept }}{\text { DS3-13 }}$} \\
\hline & DS3-5 & DS5-7 & DS7-13 & DS3-13 & \\
\hline \multicolumn{6}{|c|}{ Onset Irrelevant } \\
\hline Non-onset & 26.0 & 27.0 & 28.5 & 27.8 & 591 \\
\hline Onset & 11.0 & 24.0 & 20.8 & 20.1 & 550 \\
\hline \multicolumn{6}{|c|}{ Onset Relevant } \\
\hline Onset & 3.5 & 9.5 & 9.3 & 8.5 & 579 \\
\hline
\end{tabular}

Note-DS, display size.

related with the onset stimulus. If increasing stimulus proximity results in perceptual grouping or contour interference between display items at larger display sizes, and this, in turn, was responsible for the relatively steep slopes obtained for the irrelevant onsets at larger display sizes, then we would expect comparable search slopes when onsets are relevant for search. If, on the other hand, the increased search slope between larger display sizes for irrelevant-onset targets was due to a modulating effect of non-onsets on attentional capture by onsets, then search slopes should be shallower for relevant-onset targets at larger display sizes.

The RT and error rate data were submitted to a two-way repeated measures ANOVA, with display size $(3,5,7$, and 13) and target type (onset target present and target absent) as factors. As shown in the bottom panel of Figure 2, for the onset-relevant condition, $\mathrm{RT}$ increased with increasing display size $[F(3,21)=7.98, p<.01]$ and increased between onset target-present and target-absent conditions $[F(1,7)=16.93, p<.01]$. As shown in Table 1 , a higher error rate occurred for onset target-present responses than for target-absent responses $[F(1,7)=24.37$, $p<.01]$.

Also worth noting is the relatively flat search slope (see Figure 2 and Table 2) for the onset-relevant targets. These data suggest that subjects are capable of rapid and parallel search through both small and large displays when they are intentionally searching for an onset target.

Comparison of onset-relevant and onset-irrelevant conditions. The analysis of the onset-relevant condition provided evidence that the close proximity of the stimuli in large displays does not preclude efficient search for an onset. If new objects capture attention, regardless of their relevance, then we would expect to observe similar search slopes between the onset-irrelevant and onsetrelevant conditions.

$\mathrm{RT}$ and error rate for the two onset target-present conditions were analyzed with an ANOVA, with onset (relevant and irrelevant) as the between-subjects factor and display size $(3,5,7$, and 13$)$ as the within-subjects factor. RT increased with increasing display size $[F(3,42)=$ $31.39, p<.01]$. The effect of onset target condition was not significant $(F<1)$. More importantly, there was a significant interaction between onset target condition and display size $[F(3,42)=5.16, p<.01]$ such that the increase in RT with increasing display size was larger for targets in the onset-irrelevant condition than in the onset-relevant condition.

An analysis of simple effects yielded significant main effects for display size between each pair of adjacent displays sizes $(p s<.05)$. The effect of onset condition was not significant between small display sizes $(p>.20)$. A display size $X$ onset target condition interaction occurred between the intermediate display sizes $[F(1,14)=7.14$, $p<.05]$ but was unreliable for large sizes $[F(1,14)=$ $3.29, p<.10$ ]. The error rate for onset targets (see Table 1) did not vary between the two onset conditions, nor by display size.

These results show that, for small display sizes, an irrelevant onset captures attention. As the number of items increases from 3 to 5, RT to an onset target remains constant relative to non-onset targets and yields a search slope that does differ from onset-relevant targets. These RT effects at small display sizes replicate prior research (Jonides \& Yantis, 1988; Yantis \& Jonides, 1984) and are consistent with the predictions of an attentional priority model of onset, or new-object, capture (Hillstrom \& Yantis, 1994; Yantis \& Hillstrom, 1994). However, a new perceptual object is not always accorded attentional priority, as evidenced by the increase in RT for onset targets at larger display sizes. RT to onset targets begins to increase as display size exceeds 5 items. Furthermore, the search slope for onset-irrelevant targets differs from that observed when an onset is correlated with the target.

Our data are consistent with the interrupt threshold hypothesis, which suggests that increasing display change (in the present case, realized via an increase in display ratio) modulates the capture of attention by irrelevant onsets. Interestingly, the search for task-relevant onsets does not appear to be disadvantaged by increasing display change. The search rate for the onset-relevant targets was relatively stable across display sizes. These data would appear to suggest that goal-directed, or top-down, search mechanisms (Treisman \& Sato, 1990; Wolfe, 1994) are less susceptible to the deleterious influence of transient display change than are stimulus-driven mechanisms, such as those responsible for the generation of attentional interrupts.

\section{EXPERIMENT 2}

In Experiment 1, we varied display ratio by increasing the number of non-onset stimuli as one means of examining the influence of display change on attentional capture by new objects. In Experiment 2, we varied stimulus ratio as another means to examine the interrupt threshold hypothesis.

The complex premasks used by Miller (1989) to equate offset and onset transients between stimuli were implemented in the displays of Experiment 2. On the basis of the interrupt threshold hypothesis, we expect that search slopes for onset targets will be relatively steep for both small and large display sizes. This follows, since, in Experiment 2 , the amount of display change, via an increase 
in the stimulus ratio, was further increased relative to that in Experiment 1. Thus, we expect a further decrease in the proportion of trials on which the appearance of new objects capture attention in a stimulus-driven fashion. On the other hand, on the basis of the results of Experiment 1 , it seems likelv that the search for onset targets (i.e., onset-relevant condition) will be relatively immune to the amount of transient change associated with the non-onset objects.

The procedures employed in Experiment 2 were identical to those of Experiment 1, with the exceptions described below. As in Experiment 1, a between-subjects design was used to manipulate onset relevance (relevant or irrelevant). Target type (present or absent) and display size $(3,5,7$, or 13$)$ were manipulated within subjects. As in Experiment 1, the onset-relevant condition was included to serve as a baseline against which to evaluate attentional capture by irrelevant onsets.

\section{Method}

Subjects. Twenty-four University of Illinois undergraduate students, who had not participated in Experiment 1, served as subjects in Experiment 2. Twelve subjects ( 7 women and 5 men) participated in the onset-irrelevant condition, and 12 subjects ( 4 women and 8 men) participated in the onset-relevant condition. All subjects demonstrated adequate near and far visual acuity (minimum 20/40) with the Snellen eye chart.

Stimuli. The premask used was a block figure eight with an " $X$ " inscribed in each half of the figure. The center panels of Figure 1 illustrate the premasks used in Experiment 2 to form the stimulus display. Other than the premasks, the stimulus letters, premask configurations, and display sizes were identical to those of Experiment 1 .

\section{Results and Discussion}

Less than $2 \%$ of the observations were discarded prior to analysis using the same criteria adopted for Experiment 1. Mean RTs for correct trials (collapsed across sessions) as a function of display size are plotted by target type for the onset-irrelevant condition and the onset-relevant condition in the top and bottom panels, respectively, of Figure 3. Error rates in all experimental conditions (collapsed across sessions) are presented in Table 3. We first report the results of the separate ANOVAs conducted on the onset-irrelevant and onset-relevant data and then describe the results of the ANOVA that contrasts the RTs and error rates obtained for the onset targets in the two conditions.

Onset-irrelevant condition. The ANOVA for the onsetirrelevant condition included session ( 1 and 2), display size (3, 5, 7, and 13), and target type (present and absent) as factors. RTs were longer for Session 1 responses $[F(1,11)=57.96, p<.01]$ and showed larger increases as display size increased $[F(3,33)=10.48, p<.01]$ in Session 1 than in Session 2. As shown in Figure 3, RT increased between target-present and target-absent trials $[F(1,11)=72.59, p<.01]$ and increased with display size $[F(3,33)=85.23, p<.01]$. Display size and target type interacted with target-absent responses, showing greater increases in RT with increasing display size than with target-present responses $[F(3,33)=47.29, p<.01]$.
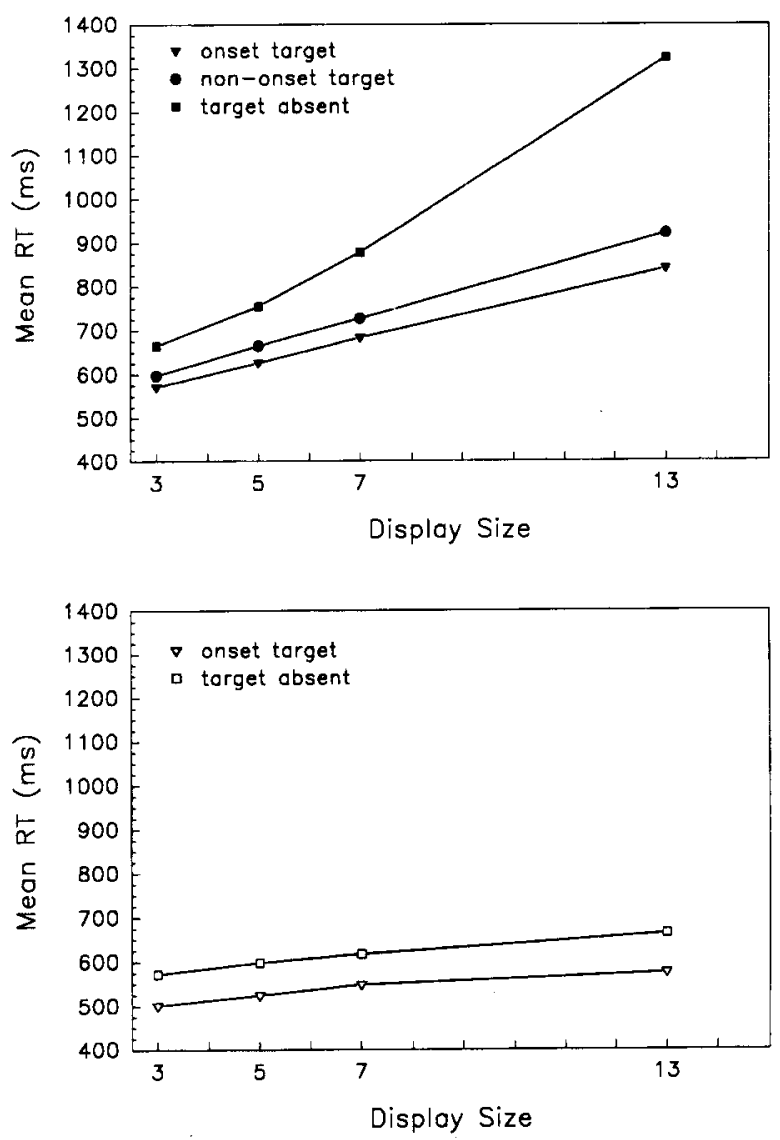

Figure 3. Mean RT (in milliseconds) by display size for onset target, non-onset target, and target-absent trials in the onsetirrelevant (top panel) and onset-relevant (bottom panel) conditions of Experiment 2.

Error rate (see Table 3) was higher for target-present responses than for target-absent responses $[F(1,11)=$ $70.82, p<.01]$, and error rate increased with display size $[F(3,33)=38.23, p<.01]$. Display size interacted with target type such that larger increases in error rate with display size occurred for target-present responses than for target-absent responses $[F(3,33)=9.28, p<.01]$.

A separate ANOVA was conducted to contrast performance in the two target-present conditions, with session (1 and 2), display size (3, 5, 7, and 13), and target type (onset and non-onset) as factors. RT decreased between Sessions 1 and $2[F(1,11)=52.32, p<.01]$. Increases in RT were observed between onset and non-onset targets $[F(1,11)=11.58, p<.01]$ and as display size increased $[F(3,33)=71.43, p<.01]$. Session interacted with display size $[F(3,33)=3.34, p<.05]$, with Session 1 responses showing larger increases in RT with increasing display size. As shown in Figure 3, the effect of target type on display size was significant $[F(3,33)=4.40, p<.01]$, with larger increases in RT as display size increased for non-onset targets than for onset targets.

An analysis of simple effects was conducted to evaluate this interaction. Main effects of display size and target type were observed between each pair of adjacent 
Table 3

Error Rate for Display Size (DS) by Target Type in the Onset-Irrelevant and Onset-Relevant Conditions of Experiment 2

\begin{tabular}{|c|c|c|c|c|}
\hline \multirow[b]{2}{*}{ Target } & \multicolumn{4}{|c|}{ Exror Rate } \\
\hline & DS3 & DS5 & DS7 & DS13 \\
\hline \multicolumn{5}{|c|}{ Onset Irrelevant } \\
\hline Absent & .03 & .03 & .04 & .05 \\
\hline Non-onset & .04 & .08 & .10 & .16 \\
\hline Onset & .04 & .06 & .06 & .07 \\
\hline \multicolumn{5}{|c|}{ Onset Relevant } \\
\hline Absent & .04 & .03 & .04 & .04 \\
\hline Onset & .05 & .06 & .07 & .05 \\
\hline
\end{tabular}

display sizes $(p s<.01)$. The display size $\times$ target interaction was not significant between smaller display sizes $(p s>.10)$. The effect of target type on display size was significant only between the two largest display sizes $[F(1,11)=11.85, p<.01]$. However, as shown in Table 4 (which provides the search slopes between adjacent display sizes), even between the large display sizes, the search slopes for both onset and non-onset targets were significantly greater than zero.

The error rate in the onset-irrelevant condition increased between onset and non-onset targets $[F(1,11)=$ $17.68, p<.01]$ and as display size increased $[F(3,33)=$ $10.71, p<.01]$. The error rate for target type also varied as a function of display size $[F(3,33)=3.97, p<.05]$ and session $[F(1,11)=9.16, p<.01]$. With increasing display size, and between Sessions 1 and 2, more errors were observed in response to non-onset targets than to onset targets.

These results show little evidence of attentional capture by irrelevant onsets and replicate Miller's (1989) results obtained at small display sizes. Consistent with the interrupt threshold hypothesis, a comparison of the search slopes for Experiments 1 and 2 for the onset-irrelevant condition (see Tables 2 and 4) also suggests that stimulus ratio and display ratio combine to modulate the ability of onset targets to capture attention in a stimulusdriven manner. A comparison of the onset-irrelevant slopes at the smaller display sizes in the two experiments provides evidence for the influence of stimulus ratio on attentional capture. Display ratio effects are observed in both experiments in the form of steep slopes for larger display sizes on the onset trials. Finally, the combined influence of stimulus and display ratio can be observed in the steeper slopes at the larger display sizes in Experiment 2 relative to those in Experiment 1.

Onset-relevant condition. As in Experiment 1, the analysis of the onset-relevant data was performed to provide a baseline against which to evaluate the rather steep search slopes obtained for the onset-irrelevant condition. The ANOVA for the onset-relevant condition included target type (target absent and onset target present) and display size as factors. RT was longer for target-absent trials than for target-present trials $[F(1,11)=9.98, p<.01]$. RT also increased with display size $[F(3,33)=44.54$, $p<.011$. Error rate was higher for target-present trials than for target-absent trials $[F(1,11)=9.20, p<.01]$.

Comparison of onset-relevant and onset-irrelevant conditions. ANOVAs for RT and error rate, with onset (relevant and irrelevant) as the between-subjects factor and display size $(3,5,7$, and 13$)$ as the within-subjects factor, were conducted to determine whether responses to an onset target differed as a function of relevance. RT increased with display size $[F(3,66)=67.00, p<.01]$ and was longer for onset-irrelevant targets than for onsetrelevant targets $[F(1,22)=9.92, p<.01]$. As suggested by Figure 3, the increase in RT with display size was greater for onset-irrelevant targets than for onset-relevant targets $[F(3,66)=22.45, p<.01]$.

An analysis of simple effects revealed main effects of display size and onset type between each pair of adjacent display sizes $(p s<.05)$. The comparisons of RT differences between adjacent display sizes indicated larger RT effects for irrelevant onsets than for relevant onsets at each comparison $(p s<.05)$. Therefore, the steep search slopes obtained for the onset-irrelevant trials cannot be explained by grouping or contour interaction phenomena. The error rate for onset targets (see Table 3) did not vary between the two onset conditions, nor by display size $(p s>.10)$.

\section{EXPERIMENT 3}

The results of Experiments 1 and 2 are consistent with the interrupt threshold hypothesis. The search rate for onset-irrelevant targets slowed with increases in the number of offset transients. Thus, it would appear that the capture of attention by new objects can be modulated by stimulus-driven factors (e.g., transient changes in old, or non-onset, objects) as well as goal-directed factors (Folk et al., 1992; Yantis \& Jonides, 1990).

Experiment 3 was conducted for two reasons. First, we were interested in determining whether a further decrease in the probability of attentional capture by new objects would occur with an increase in the stimulus ratio. To that end, non-onset premasks were increased in complexity (see Figure 1, bottom panels) such that more segments would be removed when a premask changed into a non-onset stimulus than segments would be added with the appearance of an onset stimulus. Second, the error rates, particularly for the non-onset trials with the large

Table 4

Search Slope (Milliseconds/Item) and Intercept (Milliseconds) for the Target-Present Conditions of Experiment 2

\begin{tabular}{|c|c|c|c|c|c|}
\hline \multirow[b]{2}{*}{ Target } & \multicolumn{4}{|c|}{ Search Slope } & \multirow{2}{*}{$\frac{\text { Intercept }}{\mathrm{DS} 3-13}$} \\
\hline & DS3-5 & DS5-7 & DS7-13 & DS3-13 & \\
\hline \multicolumn{6}{|c|}{ Onset Irrelevant } \\
\hline Non-onset & 34.0 & 31.0 & 32.3 & 32.3 & 502 \\
\hline Onset & 27.5 & 28.5 & 26.2 & 26.9 & 492 \\
\hline \multicolumn{6}{|c|}{ Onset Relevant } \\
\hline Onset & 11.5 & 12.0 & 4.5 & 7.1 & 487 \\
\hline
\end{tabular}

Note-DS, display size 
display sizes, were rather high in our first two experiments. We attempted to decrease the error rates in Experiment 3 by further emphasizing accurate responding. As in the first two experiments, onset relevance (irrelevant and relevant) was manipulated between subjects. Target type (present or absent) and display size were varied within subjects. The experimental design and procedures were identical to those of Experiments 1 and 2.

\section{Method}

Subjects. Twenty-four University of Illinois undergraduate students, who had not participated in the first two experiments, served as paid subjects in the Experiment 3. Twelve subjects ( 9 women and 3 men) participated in the onset-irrelevant condition, and 12 subjects ( 4 women and $8 \mathrm{men}$ ) participated in the onset-relevant condition. All subjects demonstrated adequate near and far visual acuity.

Stimuli. The bottom panels of Figure 1 illustrate the premask and stimulus displays of Experiment 3 . To increase the complexity of the premask, a four-segment "star" was inscribed in each half of the block figure eight. The size of the premask was increased to $0.96^{\circ}$ height and $0.55^{\circ}$ width such that the line segments of the premask were distinct at a viewing distance of $90 \mathrm{~cm}$. The premasks were positioned $5.0^{\circ}$ from fixation, yielding a minimum edge-toedge separation between onset and non-onset stimuli of $0.25^{\circ}$ visual angle.

\section{Results and Discussion}

Using the procedure described for the previous experiments, less than $2 \%$ of the observations were excluded from the analysis. Mean RTs for correct trials (collapsed over session) by display size for each target type are presented for the onset-irrelevant and onset-relevant conditions in the top and bottom panels, respectively, of Figure 4. Error rate is shown in Table 5. The analyses consisted of separate ANOVAs for the onset-irrelevant condition and the onset-relevant condition, followed by ANOVAs that examined the effect of onset targets in the two conditions. In addition, an ANOVA was conducted to evaluate the effect of premask complexity across the three experiments.

Onset-irrelevant condition. The ANOVAs for RT and error rate in the onset-irrelevant condition included session ( 1 and 2$)$, display size $(3,5,7$, and 13$)$, and target type (present and absent) as factors. RTs were slower in Session 1 than in Session $2[F(1,11)=55.62, p<.01]$, increased with display size $[F(3,33)=126.52, p<.01]$, and between target-present and target-absent conditions $[F(1,11)=144.04, p<.01]$. Session interacted with display size $[F(3,33)=3.47, p<.05]$ and with target type $[F(1,11)=7.64, p<.05]$, with Session 1 showing greater increases in RT with display size and between targetpresent and target-absent conditions than Session 2. Display size and target type interacted $[F(3,33)=92.01, p<$ $.01]$, with the target-absent condition showing greater increases in RT with increasing display size followed by the target-present conditions.

The error rate was higher in Session 1 than in Session 2 $[F(1,11)=5.83, p<.05]$. Errors increased with display size $[F(3,33)=15.96, p<.01]$ and between target-absent and target-present conditions $[F(1,11)=42.78, p<.01]$.
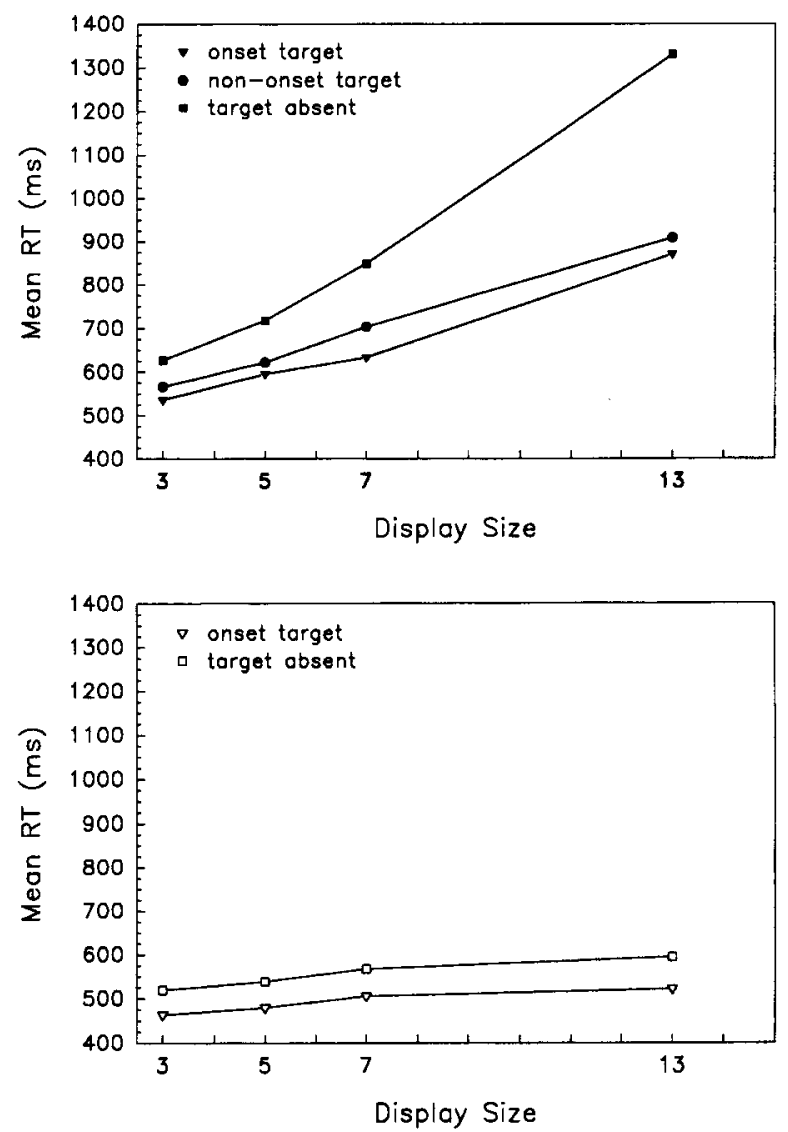

Figure 4. Mean RT (in milliseconds) by display size for onset target, non-onset target, and target-absent trials in the onsetirrelevant (top panel) and onset-relevant (bottom panel) conditions of Experiment 3.

Separate ANOVAs for RT and error rate were conducted to evaluate the two target-present conditions, with session ( 1 and 2$)$, display size $(3,5,7$, and 13), and target type (onset and non-onset) as factors. RT decreased between Sessions 1 and $2[F(1,11)=29.32, p<.01]$, increased with increasing display size $[F(3,33)=84.41, p<.01]$ and between onset and non-onset targets $[F(1,11)=25.94$, $p<.01]$. In contrast to the results of the first two experiments, the display size $\times$ target type interaction failed to reach significance $(p>.25)$.

Error rate increased between the onset and non-onset targets $[F(1,11)=27.00, p<.01]$. Display size and target type interacted $[F(3,33)=3.5, p<.05]$, with a greater increase in errors as display size increased observed for non-onset targets, relative to onset targets.

Onset-relevant condition. The ANOVA for the onsetrelevant condition included target type (present and absent) and display size as factors. RT increased with increasing display size $[F(1,11)=26.85, p<.01]$ and between target-present and target-absent conditions $[F(1,11)=31.45, p<.01]$. Error rate showed a small increase between target-absent and target-present responses $[F(1,11)=4.77, p=.05]$. 
Table 5

Error Rate for Display Size (DS) by Target Type in the Onset-Irrelevant and Onset-Relevant Conditions of Experiment 3

\begin{tabular}{lcccc}
\hline & \multicolumn{3}{c}{ Error Rate } \\
\cline { 2 - 3 } \multicolumn{1}{c}{ Target } & DS3 & DS5 & DS7 & DS13 \\
\hline \multirow{3}{*}{ Absent } & .03 & .03 & .03 & .06 \\
Non-onset & .06 & .07 & .08 & .11 \\
Onset & .04 & .07 & .05 & .06 \\
& & Onset Irrelevant & \\
Absent & .02 & .03 & .03 & .03 \\
Onset & .04 & .03 & .03 & .04 \\
\hline
\end{tabular}

Table 6

Search Slope (Milliseconds/Item) and Intercept (Milliseconds) for the Target-Present Conditions of Experiment 3

\begin{tabular}{|c|c|c|c|c|c|}
\hline \multirow[b]{2}{*}{ Target } & \multicolumn{4}{|c|}{ Search Slope } & \multirow{2}{*}{$\frac{\text { Intercept }}{\text { DS3-13 }}$} \\
\hline & DS3-5 & DS5-7 & DS7-13 & DS3-13 & \\
\hline \multicolumn{6}{|c|}{ Onset Irrelevant } \\
\hline Non-onset & 28.0 & 40.5 & 34.2 & 34.6 & 457 \\
\hline Onset & 29.5 & 19.0 & 39.5 & 33.7 & 423 \\
\hline \multicolumn{6}{|c|}{ Onset Relevant } \\
\hline Onset & 8.0 & 13.0 & 8.0 & 5.6 & 453 \\
\hline
\end{tabular}

Note-DS, display size.

Comparison of onset-relevant and onset-irrelevant conditions. ANOVAs were conducted to evaluate RT and error rate for the target-present conditions, with onset (relevant and irrelevant) as the between-subjects factor and display size as the within-subjects factor. RT increased between onset-relevant and onset-irrelevant targets $[F(1,22)=32.82, p<.01]$ and with increasing display size $[F(3,66)=57.65, p<.01]$. The increase in $\mathrm{RT}$ as a function of display size was larger for onset-irrelevant targets than for onset-relevant targets $[F(3,33)=30.55, p<.01]$.

Analysis of simple effects yielded main effects of display size and onset type between each pair of adjacent display sizes $(p s<.01)$. As suggested by the search slopes given in Table 6 , the analysis also revealed that, relative to onset-relevant targets, larger increases in RT occurred for onset-irrelevant targets between small ( 3 and 5) display sizes and large (7 and 13) display sizes $(p s<.01)$, but not for intermediate display sizes $(p>.3)$. The error rate for onset targets (see Table 5) did not vary between the two onset conditions, nor by display size ( $p$ s > .3).

Analysis of the premasks. To evaluate the effect of increasing premask complexity, an ANOVA was conducted using premask condition as the between-subjects variable (i.e., between Experiments 1, 2, and 3) and using target type (onset and non-onset) and display size as the within-subjects variables. Significant main effects for RT were observed for display size $[F(3,87)=186.12, p<.01]$ and target type $[F(1,29)=72.24, p<.01]$. Target type interacted with display size $[F(3,87)=7.38, p<.01]$ and premask condition $[F(3,87)=4.48, p<.05]$. The threeway interaction $[F(6,87)=2.38, p<.05]$ was evaluated in separate analyses for onset and non-onset targets. For non-onset targets, only a main effect of display size was observed $[F(3,87)=218.26, p<.01]$. For onset targets, a main effect of display size $[F(3,87)=109.05, p<.01]$ and a display size $\times$ premask interaction $[F(6,87)=2.87$, $p=.01]$ were observed. The display size slope for onset targets increased from $20 \mathrm{msec}$ for the simple figure eights (Experiment 1), to $27 \mathrm{msec}$ for the figure eights with the inscribed " $X$ " (Experiment 2), to $34 \mathrm{msec}$ for the complex premasks (Experiment 3 ).

Errors decreased as premask complexity increased from the simple figure-eight premasks to the complex premasks of Experiment $3[F(2,29)=3.73, p<.05]$, increased with increasing display size $[F(3,87)=18.07, p<$ $.01]$ and between onset and non-onset targets $[F(1,29)=$ $46.33, p<.01]$. A larger increase in error as a function of display size was observed for non-onset targets, relative to onset targets $[F(3,87)=4.45, p<.01]$.

\section{GENERAL DISCUSSION}

The results of the three experiments, which involved two different manipulations of display change (i.e., display ratio and stimulus ratio), are consistent with the interrupt threshold hypothesis. This hypothesis suggests that capture of attention by new objects will become less likely as the amount of (non-onset) change in the visual field increases. The reduction in capture is attributed to a fixed ratio threshold that must be exceeded for the attentional interrupt - and, hence, attentional capture by the new object-to be realized. That is, it is assumed that (non-onset) display change produces noise in the interrupt mechanism, and, with increasing noise, it becomes less likely that the appearance of a new object will produce sufficient activation such that the fixed ratio threshold will be exceeded, thereby triggering an attentional interrupt. However, even in the absence of the production of a stimulus-driven attentional interrupt, the new object can receive preferential treatment in visual search. This was demonstrated by the results obtained in the onsetrelevant conditions in which subjects intentionally searched for the new object. Across the three experiments, search rates were fast and relatively insensitive to the magnitude of display change.

The results of Experiment 1 revealed that when the appearance of an onset stimulus is not correlated with target identity or location, RT to onset targets is relatively independent of display size when there are relatively few display items (3-5 stimuli). This finding replicates prior research providing evidence that onsets capture attention. However, as display size increased (from 5 to 13 stimuli) with a corresponding increase in the display ratio of non-onsets to onsets, RT to an onset target also began to increase.

In Experiment 2, we used the complex premasks of Miller (1989) such that the number of offset stimulus transients and the number of onset stimulus transients were approximately equal, in order to examine the extent to which both the stimulus ratio and the display ratio limit capture by irrelevant onsets. Our results at small display sizes correspond to those of Miller: Offset transients re- 
Table 7

Observed and Predicted Mean RT (Milliseconds) for the Strong and Weak Capture Models

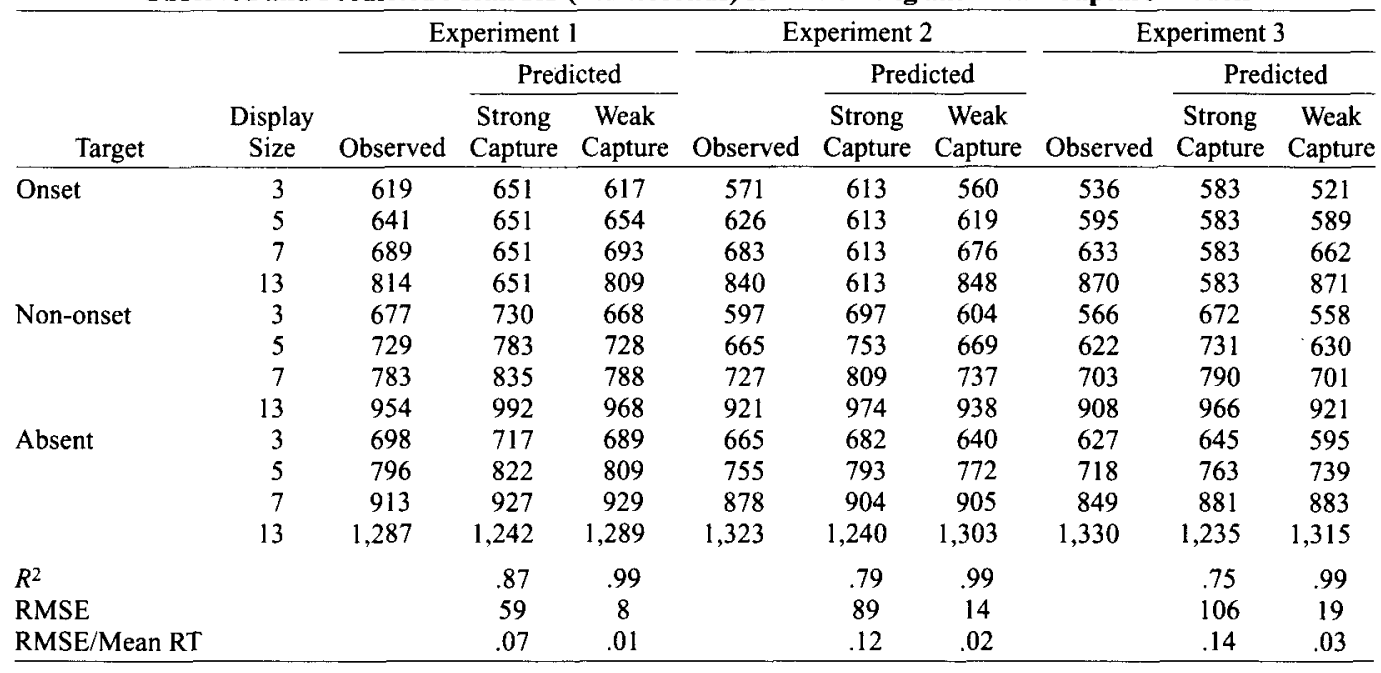

duced the probability of attentional capture by onset transients. In addition, the results of Experiment 2 show that stimulus ratio further decreased the rate at which the subjects identified onset-irrelevant targets in large displays relative to the findings obtained in Experiment 1. Finally, in Experiment 3, in which the number of offset transients exceeded the number of onset transients at the level of the single stimulus, the rate at which onsetirrelevant targets were identified was slowed even further.

Collectively, these results provide strong support for the interrupt threshold hypothesis. However, thus far, we have offered only qualitative support for this hypothesis. While the changes in search slope for the onset-irrelevant targets are in agreement with the predictions of the interrupt threshold hypothesis, our proposal would be strengthened by a quantitative demonstration that the proportion of trials on which onsets capture attention declines with increasing display change.

In an effort to provide such support, we fit the data obtained in the three experiments to two different quantitative models of attention capture. Both of these models have been previously used by Yantis and Jonides to characterize attentional capture (Jonides \& Yantis, 1988; Yantis \& Jonides, 1984). In one model, which we will call the strong capture model, it is assumed that if an onset item is present, it is identified first; otherwise, search occurs in a serial self-terminating fashion through the objects in the display. In the second model, which we will call the weak capture model, it is assumed that attentional capture by new objects may fail on some proportion of trials. On trials on which capture does not occur, the display is searched in a serial fashion for the target.

Since the interrupt threshold hypothesis predicts that the probability that new objects will capture attention will decrease with increases in the magnitude of display change, we expect that (1) the goodness of fit for the strong capture model will decrease from Experiment 1 to Experi- ment 2 and, finally, to Experiment 3 and (2) for the weak capture model, the estimate of the proportion of trials on which attentional capture by new objects occurs should decrease from Experiment 1 to Experiment 2 and, finally, to Experiment 3.

The data presented in Table 7 address these predictions. Consistent with the interrupt threshold hypothesis, the amount of variance accounted for (i.e., $R^{2}$ ) by the strong capture model declined with an increase in stimulus ratio. As the non-onset premasks became more complex-resulting in an increasing number of offset transients - the variance accounted for by the strong capture model declined from $87 \%$ to $75 \%$. Similarly, the root mean square error (RMSE) increased with increasing stimulus ratio. As can be seen in Table 7, the weak capture model fit the data from the three experiments equally well. However, what is not reflected in the table is that this was accomplished by an adjustment in the parameter, which considers the number of trials on which new objects captured attention. This parameter was estimated as $64 \%, 49 \%$, and $40 \%$ for Experiments 1,2 , and 3 , respectively. Thus, as predicted by the interrupt threshold hypothesis, the proportion of trials on which attentional capture by new objects was estimated to occur declined with increasing stimulus ratio (i.e., with increases in the number of offset transients). ${ }^{1}$

The interrupt threshold hypothesis also suggests that the proportion of trials on which capture occurs should vary with display ratio, at least when relatively simple non-onset premasks are employed. To examine this prediction, we fit the strong capture model separately to the mean RT data obtained at the large and small display sizes in Experiment 1. Consistent with our hypothesis, the variance accounted for by the strong capture model was substantially higher for the small display sizes $(98 \%)$ than for the large display sizes $(86 \%)$. Additionally, the estimate of the proportion of trials on which attention was captured by the onset target decreased from the small 
display sizes to the large display sizes. These data suggest that the probability of attentional capture by new objects decreases with increasing numbers of non-onset objects. ${ }^{2}$

Finally, assuming that top-down, or goal-directed, control of attention is relatively insensitive to the magnitude of display change, we would expect that the onset-relevant data from Experiments 1, 2, and 3 would be well fit by the strong capture model. The variance accounted for by the strong capture model was $97 \%, 99 \%$, and $98 \%$ for Experiments 1, 2, and 3, respectively. Therefore, it would appear that, although stimulus-driven attentional capture is modulated by display change in the form of offset transients, the intentional search for a new-object target is relatively immune to changes in at least some physical characteristics of objects in the visual field.

Similar limitations upon stimulus-driven attentional capture occur in response to goal-directed, or top-down, factors. When attention is focused in response to central cues, irrelevant peripheral onsets fail to elicit capture (Koshino, Warner, \& Juola, 1992; Theeuwes, 1991; Yantis \& Jonides, 1990). Conversely, irrelevant feature singletons may capture attention, given a goal-directed attentional control setting for a particular feature (Folk et al., 1992) or when subjects engage in singleton detection mode (Bacon \& Egeth, 1994).

On the basis of our results, we suggest that, in addition to goal-directed factors such as those described above, stimulus-driven attentional capture may be modulated by salient features (i.e., offset transients) of display stimuli, which, like onsets, are irrelevant to target location or identity but nonetheless interfere with the generation of attentional interrupts. The allocation of attention to a particular element is then not solely determined by its status as a new object, but it can also be limited by the demands of other objects and events within the visual field.

\section{REFERENCES}

BACON, W. F., \& EgETH, H. E. (1994). Overriding stimulus-driven attentional capture. Perception \& Psychophysics, 55, 485-496.

Folk, C. L., REMing TON, R. W., \& JoHnston, J. C. (1992). Involuntary covert orienting is contingent on attentional control settings. Journal of Experimental Psychology: Human Perception \& Performance, 18, 1030-1044.

Hillstrom, A. P., \& YANTIS, S. (1994). Visual motion and attentional capture. Perception \& Psychophysics, 55, 399-411.

JONIDES, J., \& YANTIS, S. (1988). Uniqueness of abrupt visual onset in capturing attention. Perception \& Psychophysics, 43, 346-354.

Koshino, H., Warner, C. B., \& Juola, J. F. (1992). Relative effectiveness of central, peripheral, and abrupt-onset cues in visual attention. Quarterly Journal of Experimental Psychology, 45A, 609-631.

MilLER, J. (1989). The control of attention by abrupt visual onsets and offsets. Perception \& Psychophysics, 45, 567-571.

Theeuwes, J. (1990). Perceptual selectivity is task dependent: Evidence from selective search. Acta Psychologica, 74, 81-99.

TheEuwes, J. (1991). Exogenous and endogenous control of attention: The effect of visual onsets and offsets. Perception \& Psychophysics, 49, 83-90.

TodD, S., \& Kramer, A. F. (1994). Attentional misguidance in visual search. Perception \& Psychophysics, 56, 198-210.

Treisman, A., \& Sato, S. (1990). Conjunction search revisited. Journal of Experimental Psychology: Human Perception \& Performance, $16,459-478$.
WoLFE, J. M. (1994). Guided Search 2.0: A revised model of visual search. Psychonomic Bulletin \& Review, 1, 202-238.

YANTIS, S., \& Hillstrom, A. P. (1994). Stimulus-driven attentional capture: Evidence from equiluminant visual objects. Journal of Experimental Psychology: Human Perception \& Performance, 20, 95-107.

YANTIS, S., \& JohnSON, D. N. (1990). Mechanisms of attentional priority. Journal of Experimental Psychology: Human Perception \& Performance, 16, 812-825.

YANTIS, S., \& JoNES, E. (1991). Mechanisms of attentional selection: Temporally modulated priority tags. Perception \& Psychophysics, 50, 166-178.

YANTIS, S., \& JoNIDES, J. (1984). Abrupt visual onsets and selective attention: Evidence from visual search. Journal of Experimental Psychology: Human Perception \& Performance, 10, 601-620.

YANTIS, S., \& JoNides, J. (1990). Abrupt visual onsets and selective attention: Voluntary versus automatic allocation. Journal of Experimental Psychology: Human Perception \& Performance, 90, 121-134.

\section{NOTES}

1. Given that the error rates differed rather dramatically between the irrelevant onset and non-onset target conditions at the larger display sizes in Experiments 1 and 2, one might question the fits of the strong and weak capture models for these data. In an effort to examine the influence of differential error rates on the model fits, we conducted a second fit of the strong and weak capture models to the mean RT data obtained in Experiments 1 and 2. In this fit of the models, we adjusted the mean RTs obtained in the non-onset target trials by decreasing the search rate by $20 \%$ to compensate for the higher error rates on the nononset target trials than on the onset target trials.

The strong capture model fit the adjusted RT data more poorly than it did the unadjusted data (i.e., the mean RT data displayed in Table 7). The strong capture model accounted for $84 \%$ and $75 \%$ of the variance in the adjusted mean RT data in Experiments 1 and 2; it accounted for $87 \%$ and $79 \%$ of the variance in the unadjusted mean RT data in the same experiments. Furthermore, the estimates of the percent of trials on which the onset targets captured attention declined from that obtained on the unadjusted mean RT data (i.e., when the weak capture model was fit to the adjusted non-onset RT data). The estimates of the proportion of trials on which the onset targets captured attention were $54 \%$ and $36 \%$ in Experiments 1 and 2, respectively. These estimates can be contrasted with estimates of $64 \%$ and $49 \%$ capture for the unadjusted mean RT data in Experiments 1 and 2, respectively.

Therefore, on the basis of these data, it appears safe to conclude that we were quite conservative in our estimation of attention capture failures in response to offset transients.

2. We have argued that attentional capture by new objects is modulated by the amount of change in old (non-onset) objects that occurs in the visual field. Furthermore, we have suggested that this process of modulation of attentional capture is the result of increasing noise in the attentional interrupt mechanism, which results in the failure of the new object to produce sufficient activation to exceed a fixed ratio threshold (i.e., the interrupt threshold hypothesis). Our data, as well as those of Miller (1989), are quite consistent with this proposal.

However, data have also been obtained in a number of studies that may appear, at first glance, to belie our interrupt threshold account. For example, Jonides and Yantis (1988; see also Yantis \& Jonides, 1984) have conducted studies in which subjects were initially presented with six figure-eight premasks (e.g., their Experiment 2). In the display size 7 condition, segments of the six premasks were removed to reveal letters, and an additional onset letter appeared in the display. However, in the display size 3 condition, four premasks disappeared completely, segments were removed from the remaining premasks to create letters, and an onset appeared in a previously unoccupied display location. For the onset targets, RTs were faster for the smaller display size than for the larger display size.

Given the larger amount of display change in the smaller display size condition in this study, one might imagine that our interrupt threshold account would predict less attentional capture by the new object for the small display size than for the large display size. However, such a prediction would clearly be inconsistent with the data in this study given the faster RT obtained in the smaller display size condition. 
What then is the solution to this apparent discrepancy between the data from disappearing premask studies and our interrupt threshold hypothesis? Miller (1989) proposed a possible solution to this conundrum that, with a slight modification, seems quite plausible in the present circumstances. Miller suggested that "offset transients do not attract attention to a region in visual space, but rather attract attention to a stimulus object. When no stimulus object remains, offset transients might have no distracting effect" (Miller, 1989, p. 570).

We suggest that display change contributes to the noise distribution in the interrupt mechanism only when a representation of an old (nononset) object is available. In the absence of the availability of an object representation, as would be the case when a premask completely disappears, display change does not influence the interrupt mechanism and, hence, has no effect on the probability of attentional capture by new ob- jects. Such a proposal that noise accrues only from display change associated with object representations would appear plausible given the fact that feature and object maps, which form the basis of many theories of visual search (Treisman \& Sato, 1990; Wolfe, 1994), represent only stimuli that are currently available to perception.

In summary, if we assume that the accrual of noise results from changes in currently available object representations, then our interrupt threshold hypothesis provides a good account of both disappearing premask and premask change studies. Of course, this assumption should be explicitly examined in additional studies.

(Manuscript received July 6, 1995;

revision accepted for publication August 13, 1996.) 